\title{
Sentidos y potencialidades del registro (auto)etnográfico en la investigación biográfico-narrativa
}

\author{
Jonathan Aguirre \\ Universidad Nacional de Mar del Plata - Argentina \\ Luis Porta \\ Universidad Nacional de Mar del Plata - Argentina
}

\section{Resumen}

Este artículo aborda los sentidos y las potencialidades epistémico-metodológicas que asume el registro autoetnográfico en la investigación biográfico-narrativa a partir de los relatos hilvanados por parte del investigador social en el transcurrir del trabajo de campo de una investigación cualitativa sobre políticas públicas de formación docente. Mediante la utilización del registro autoetnográfico, se evidencia que la autoetnografía narrativa a la vez que posibilita reconstruir la experiencia de los sujetos investigados mediante de sus propios relatos, implica emocionalmente al investigador social desde sus propias sensibilidades y subjetivaciones, objetivándolas y validándolas en el proceso de interpretación de los datos recopilados en la investigación.

Palabras clave: Autoetnografía. Investigación biográfico-narrativa. Reflexividad. Investigación social.

\section{Senses and potentialities of the (auto)ethnographic re- cord in the biographic-narrative research.}

\begin{abstract}
This article deals with the senses and the epistemic-methodological potentials that the autoethnographic record assumes in biographical-narrative research based on the stories researched by the social researcher in the course of the field work of a qualitative research on teacher education public policies. . Using the autoethnographic record, it is evident that narrative autoethnography, while enabling, reconstructing the experience of the subjects investigated through their own stories, emotionally implicates the social researcher from their own sensitivities and subjectifications, objectifying and validating them in the process of interpretation of the data collected in the investigation.
\end{abstract}

Keywords: Autoethnography, Biographical-narrative research. Reflexivity. Social investigation 


\section{Sentidos e potencialidades do registro (auto)etnográfico na pesquisa biográfica-narrativa}

\section{Resumo}

Este artigo trata dos sentidos e das potencialidades epistemológico-metodológicas que o registro autoetnográfico assume na pesquisa biográfico-narrativa a partir das histórias pesquisadas pelo pesquisador social no decorrer do trabalho de campo de uma pesquisa qualitativa sobre políticas públicas de formação docente.Utilizando o registro autoetnográfico, fica evidente que a autoetnografia narrativa, ao possibilitar reconstruir a experiência dos sujeitos investigados por meio de suas próprias histórias, implica emocionalmente o pesquisador social a partir de suas próprias sensibilidades e subjetivações, objetivando-as e validando-as no processo de interpretação dos dados recolhidos na investigação.

Palavras-chave: Autoetnografia. Pesquisa biográfica-narrativa. Reflexividade. Pesquisa social.

\section{Les sens et les potentialités de l'enregistrement ethnogra- phique (auto) dans la recherche biographique-narrative}

\section{Résumé}

Cet article traite des sens et des potentialités épistémologiques et méthodologiques que revêt le dossier auto-ethnographique dans la recherche biographique-narrative basée sur les histoires étudiées par le chercheur social dans le cadre d'une recherche qualitative sur les politiques publiques de formation des enseignants. . En utilisant le dossier autoethnographique, il est évident que l'autoethnographie narrative, tout en permettant de reconstruire l'expérience des sujets étudiés à travers leurs propres histoires, implique émotionnellement le chercheur social de leurs propres sensibilités et subjectivations, les objectivant et les validant dans le processus d'interprétation des données collectées dans l'enquête.

Mots-clés: Autoethnographie. Recherche biographique-narrative. Réflexivité. Recherche sociale.

\section{Introducción}

Como sucede actualmente con muchos de los conceptos que suelen utilizarse en las ciencias sociales en general, y en la investigación cualitativa en particular, éstos se han vuelto polisémicos, es decir, cuentan con una pluralidad de significados. El término etnografía no es la excepción ya que su sentido no sólo se ha ido modificando, sino 
que ha ido haciéndose más complejo (Blanco, 2012). El método etnográfico, desde la primera sistematización de sus reglas básicas que realizara el antropólogo B. Malinowski a principios del siglo XX, se ha ido enriqueciendo de forma progresiva de la práctica y la experiencia de campo hasta alcanzar en la actualidad elevadas cuotas de reconocimiento en el ámbito de las ciencias sociales y humanas (Guerrero Muñoz, 2014). No es el objetivo del presente artículo dar cuenta de la historia de lo que a lo largo de poco más de un siglo se ha ido entendiendo por el término etnografía, además de que existe una amplia gama de textos que abordan su desarrollo (Agar, 2006 y Stoller, 1999). Lo que sí es posible advertir es que a partir de dicho crecimiento y la permanente búsqueda de nuevas respuestas e indagaciones, la autoetnografía se transforma en una modalidad cualitativa potente para el abordaje de los procesos subjetivos que les suceden a los sujetos investigados y al propio investigador en las recurrentes y entramadas etapas de una investigación (Noy, 2003).

La autoetnografía se convierte así en enfoque de investigación y escritura que busca describir y analizar sistemáticamente la experiencia personal con el fin de comprender la experiencia cultural.

Esta aproximación desafía las formas canónicas de hacer investigación y de representar a los otros, a la vez que considera a la investigación como un acto político, socialmente justo y socialmente consciente. Para hacer y escribir autoetnografía, el investigador aplica los principios de la autobiografía y de la etnografía. Así, como método, la autoetnografía es, a la vez, proceso y producto (Ellis, Adams y Bochner, 2015, p.250).

A diferencia de otros formatos autorreferenciales, la autoetnografía, utilizando los materiales autobiográficos del investigador como datos primarios, enfatiza el análisis cultural y la interpretación de los comportamientos de los investigadores, de sus pensamientos y experiencias, habitualmente a partir del trabajo de campo, en relación con los otros. De esta forma, los autoetnógrafos reconocen las innumerables maneras en que la experiencia personal influye y transforma el proceso mismo de investigación (Denzin, 2017) reconociendo y dando lugar a su propia subjetividad, emocionalidad e implicancia en el trabajo de investigación (Ellis, Adams y Bochner, 2015).

Por tanto, en este artículo, particularmente, nos proponemos reflexionar sobre las potencialidades metodológicas y epistemológicas que asume el registro autoetnográfico en una investigación biográfico-narrativa a partir de los sentidos y relatos que fuimos hilvanando en el transcurrir del trabajo de campo de nuestra investigación sobre políticas públicas de formación docente. La misma forma parte de una investigación doctoral mucho más amplia desarrollada en el Centro de Investigaciones Multidisciplinarias en Educación (CIMED) de la Universidad Nacional de Mar del Plata y es parte integral de las investigaciones que realiza desde el año 2003 el Grupo de Investigaciones en Educación y Estudios Culturales (GIEEC) relacionadas al enfoque biográfico-narrativo en la formación del profesorado, las identidades docentes, las políticas públicas de formación y las pasiones y emociones puestas en juego en los relatos de profesores universitarios en relación a la educación superior (Álvarez, Porta y Sarasa, 2010; Porta y Yedaide 2017; Porta, Aguirre y Bazán, 2017). 
A los efectos de validar metodológicamente nuestras reflexividades y experiencias en campo, diseñamos un instrumento de investigación: el diario (auto)etnográfico. Concebimos este registro como una auto-narración de las propias vivencias y reflexividades del investigador a lo largo del proceso investigativo. Un diario personal que se transforma en documento narrativo en la medida que lo objetivamos y al volver sobre la narración, le otorgamos diversas significaciones y múltiples sentidos (Suarez, 2017).

La escritura de nuestros sentimientos, decisiones, afecciones, permitió una recursividad y una cristalización de significaciones que nos posibilitaron habitar la investigación desde otras miradas y reflexionar a partir de las mismas. Por esta razón sostenemos que la escritura sobre uno mismo, es decir, el relato autobiográfico, posee un valor altamente formativo,

El darse cuenta de las emociones y sentimientos, de las creencias e ideologías que la experiencia vivida despierta en el recuerdo del entorno y las circunstancias, en el pasaje a la palabra escrita, en las relecturas sucesivas que permiten modificaciones, en esa hechura cuidadosa, comprometida, implicada está buena parte del potencial formativo de la narración (Souto, 2016, p. 43).

Como se puede apreciar, el enfoque biográfico-narrativo viene a constituirse en camino y posibilidad de brindarle al investigador una suerte de catalizador de sus propios sentimientos, reflexividades y significaciones durante todo el proceso de trabajo de campo, y en la misma medida habilita la recuperación de las voces y las vivencias de quienes forman parte del universo a indagar. La narrativa, tanto del investigador como de los sujetos, es central para interpretar y comprender el fenómeno social en su complejidad (Marques y Satriano, 2017).

Presentaremos a continuación los aspectos metodológicos de la investigación distinguiendo las diversas etapas que asumió el trabajo de campo. Luego nos centraremos en las potencialidades metodológicas de la autoetnografía en el complejo mundo de las investigaciones biográfico-narrativas y finalizaremos el artículo habilitando el debate a partir de las conclusiones del trabajo interpretando a la narrativa (auto)etnográfica como magma de significaciones y cristalización de sentidos.

\section{Aspectos metodológicos de la investigación: Referencias a las etapas del trabajo de campo.}

El presente apartado tiene como finalidad contextualizar la investigación de la cual parte el registro autoetnográfico que aquí se presenta, como también situar al lector en las diversas etapas del trabajo de campo realizado en el marco de la tesis doctoral "La formación docente en Argentina. Un estudio interpretativo de las políticas nacionales. El caso de los denominados polos de desarrollo (2000-2001)".

En el año 2000 se inició la política denominada "Polos de Desarrollo" en el marco del "Programa Nacional de Formación Docente" del Ministerio de Cultura y Educación 
de la Nación Argentina, coordinado por Edith Litwin y su grupo de trabajo. El proyecto en cuestión tuvo como principal objetivo "el fortalecimiento y el enriquecimiento de la formación docente y de las instituciones formadoras" (Aguirre, 2017). A partir de estimular propuestas educativas innovadoras y con alto recupero pedagógico, se propuso potenciar la formación de los futuros maestros proponiendo redes de trabajo colaborativo entre las instituciones educativas regionales a través de líneas de especialización que los propios actores locales escogían. Para la implementación del proyecto se seleccionaron 84 Institutos de formación de profesores de todo el país que trabajaron en red con instituciones asociadas: una universidad nacional mediante alguna unidad académica (una cátedra, un departamento, un instituto o una facultad); más de una escuela, todas ellas próximas geográficamente al polo, pero de características sustantivamente diversas como campo de acción educativa, y otros institutos en una acción concertada con las jurisdicciones (Informe de Gestión. Diciembre 2001. MCyE de la Nación Argentina).

En este contexto nuestra investigación intenta recuperar las potencialidades y tensiones de dicha política pública no desde un abordaje clásico en donde las normativas y los documentos oficiales son el centro del análisis, sino que partimos de comprender las políticas públicas como entramados puestos en acción en el territorio a través de sujetos que se apropian, resisten, resignifican los diversos sentidos de la política pública en cuestión (Porta y Aguirre, 2017; Gvritz y Torre 2015). Al analizar la política de Polos de Desarrollo, nos centramos en las narrativas de aquellos actores que han sido parte de la experiencia desde diversas responsabilidades e implicaciones. Sus relatos nos brindan la posibilidad de inmiscuirnos en un territorio poco abordado en la investigación sobre políticas públicas en Argentina y que está sujeto a los sentimientos, experiencias, vivencias de los sujetos que intervinieron en la puesta en marcha de la política. La narrativa nos otorga la oportunidad de habitar esos relatos e interpretar el proyecto Polos de Desarrollo desde nuevas miradas. A los efectos de recuperar esta experiencia, realizamos estancias intensivas de trabajo en Institutos de Formación Docente que fueron sede del proyecto relacionándonos con los actores que fueron parte de la experiencia.

El trabajo de campo de nuestra investigación comenzó antes de visitar las instituciones formadoras seleccionadas. Para comprender la política pública del Ministerio de Educación nacional, en primer lugar entrevistamos a todos los coordinadores y al equipo nacional del proyecto Polos de Desarrollo. Para ello se utilizó la técnica de entrevista semi estructurada. En ellas se adoptó el enfoque (auto)biográfico-narrativo como forma de indagación en la realidad educativa (Denzin y Lincoln, 2015). El objetivo primordial de este recurso fue captar las narraciones en el medio más natural posible. Dentro del tipo de entrevista en profundidad, se adoptó "la forma estandarizada, abierta, de carácter semiflexible con un guion de preguntas que se hacen a todos los entrevistados, aunque no exactamente en el mismo orden" (Taylor y Bogdan 2007, p.100). El carácter de semi-abiertas de estas entrevistas dio lugar a que el entrevistado adquiriera un rol más activo, por momentos casi guiando la entrevista mediante una reconstrucción hilvanada de sucesos del pasado en función de su relación con el presente y con el proyecto (Bolívar, 2016). 
De dichas entrevistas - 7 en total — surgieron los Institutos de Formación Docente que decidimos indagar, ya que en los relatos de los entrevistados, dichos establecimientos emergían como espacios en donde la política había producido actividades y experiencias pedagógicas formativas, tanto para los estudiantes de los profesorados, como para los docentes de los mismo. Aquí es cuando se hace evidente que el mismo campo va moldeando la investigación y la va llevando por caminos que a priori no eran previstos (Gessaghi, 2016). Se trabajó con 4 (cuatro) Institutos de Formación Docente, - los Institutos de Formación Docente N803 de Puerto Madryn (Chubut), la Escuela Normal José Gorostiága de la Banda (Santiago del Estero), el Instituto N³1 de Necochea (Buenos Aires), el Instituto N³5 Vicente D`Abramo de Monte Grande (Buenos Aires).

Al tiempo que entrevistamos a los coordinadores nacionales del proyecto, y antes de visitar las instituciones, se realizaron entrevistas a especialistas del campo de la Formación Docente con extensa trayectoria académica y gran incidencia en la producción de conocimiento del objeto de estudio. Esto permitió, en primer lugar, tener el testimonio de los referentes de la formación docente con respecto a la política coordinada por Edith Litwin y, en segundo lugar, nos posibilitó comenzar la instancia del trabajo de campo en las instituciones con un panorama amplio y a la vez complejo del Proyecto Polos de Desarrollo${ }^{1}$.

Cabe aclarar que las entrevistas están metodológica y éticamente validadas a partir de un consentimiento informado que cada uno de los entrevistados firma luego de revisar la desgrabación de la conversación establecida. Este consentimiento, junto con las voces narradas de los protagonistas, le otorgan a la investigación una doble validación metodológica y ética.

Así, nuestro trabajo de campo consistió en viajes y visitas a los Institutos, Sede del proyecto Polos de Desarrollo seleccionados, en donde se utilizaron diversas técnicas e instrumentos de recolección de datos. Desde el mes de junio de 2016 hasta octubre de 2017, se realizaron entrevistas en profundidad a los miembros del Equipo Polos de Desarrollo, a los directivos de los Institutos, a estudiantes, se pudieron llevar a cabo grupos focales, se alcanzó un registro documental de más de 200 fotos de las diversas producciones y actividades que realizaron los Institutos y paralelamente a todas las visitas se confeccionó un diario de campo en donde fuimos registrando todo lo acontecido en el terreno de la investigación² (Fernández, 2010).

Asimismo, durante las diversas instancias en los institutos, realizamos trabajo de archivo en donde se recuperaron producciones del proyecto Polos de Desarrollo de cada institución. En este punto, nos parece interesante destacar la recuperación de la perspectiva de hacer "Etnografía de Archivo" (Rockwell, 2009, p. 157) El desafío que nos presenta abordar los archivos documentales desde una perspectiva etnográfica

1 Se realizaron 5 entrevistas a especialistas académicos reconocidos por sus pares en el ámbito universitario y de gestión educativa en diversos niveles estatales.

2 Al finalizar el trabajo de campo se contó con más de 40 entrevistas realizadas a diversos actores relacionados con la política indagada. Se pudieron confeccionar 4 Grupos Focales, se recogieron mas de 250 registros fotográficos y se revisaron mas de 20 documentos oficiales institucionales en referencia al proyecto Polos de Desarrollo. 
es justamente poder imaginar la historia no escriturada de las instituciones de nuestra investigación. De esta forma, lo que enriquece el análisis etnográfico de las fuentes oficiales y normativas es leer el discurso sobre la práctica como práctica de un discurso (Rockwell, 2009).

La codificación de los diversos fragmentos que se presentarán a continuación se realizó en base a las fechas y lugares de su registro por parte del investigador. Cada una de las hojas del diario autoetnográfico fueron codificadas con una numeración. De esta manera se referenciará a los fragmentos narrativos con el siguiente código: Registro Autoetnográfico (RA No...., lugar y fecha).

Las reflexiones y narrativas que a continuación se explicitan se desprenden de las experiencias vividas desde aquellas primeras entrevistas realizadas a los coordinadores nacionales del proyecto Polos de Desarrollo, hasta las últimas visitas a los institutos superiores del conurbano bonaerense. Contextualizamos los registros autoetnográficos presentados puesto que "no se debe mantener un discurso local narrativo y biográfico, divorciado de la comprensión del contexto social e histórico y de los procesos sociales que viven los sujetos" (Goodson, 2017, p. 90). Desde esta lógica, las narrativas pueden expresar todo su potencial cuando están plenamente situadas en su tiempo y lugar (Goodson, 2017).

\section{Potencialidades teóricas-metodológicas de los registros autoetnográfi- cos en la investigación biográfico- narrativa.}

Como sostuvimos en líneas anteriores, entendemos al proceso de investigación como un proceso reflexivo permanente. Dicho proceso posibilita darnos cuenta y dar cuenta de nuestras propias acciones, decisiones y de la diversidad de condicionantes que las determinan. Así, la reflexión permite tomar en cuenta la relación entre teoría y práctica, entre pensamiento y acción (Sanjurjo, 2002). A continuación, se presentan dos retazos narrativos que evidencian las reflexiones que el investigador va hilvanando de acuerdo avanzan las entrevistas. Microdecisiones que se objetivan gracias al registro narrativo (auto)etnográfico.

"La entrevista que acabo de hacer fue la más difícil que hice hasta el momento. Siento que ambos, entrevistador y entrevistado nos probamos, nos medimos hasta donde estaba dispuesto a indagar y a responder cada uno. Fue la entrevista más difícil, pero a la vez la que más me enseñó porque ni bien comenzamos la entrevistada dejo en claro su postura y su posicionamiento. Eso me enseñó que explicitar la estructura del guión de preguntas antes de grabar es central para generar confianza en el entrevistado, y saber hasta dónde está dispuesto a narrar y al mismo tiempo aprendí que la sinceridad y humildad son la clave de todo encuentro entre sujeto que investiga y sujeto investigado, porque fue desde esos dos lugares que pudimos encontrarnos" (RA N54-55. Ciudad de Córdoba, 28-04-2017)

"Por un momento tuve ganas de cerrar la entrevista porque sentía que no respondía con comodidad y que sus respuestas no iban a aportar en demasía a la reconstrucción de la experiencia. Después recordé mi ansiedad y que debía darle tiempo y darme tiempo para 
pensar estrategias metodológicas que ayuden a la entrevistada a recordar su experiencia y a generar el clima propicio para que pueda liberarse (...) Luego de ese momento, decidí preguntar cuestiones relacionadas al trabajo en equipo durante el proyecto y cuestiones más vinculares. Allí ella se sintió más cómoda y relajada y pudo explayarse mucho más. Al cerrar la entrevista me fui con la sensación de haber tomado decisiones concretas en el medio de la entrevista y el poder darme el tiempo y esperar" (RA N44. Ciudad de Buenos Aires, 2903-2017)

El punto nodal de la reflexividad es considerar al hombre como parte del mundo social, interactuando, observando y participando con otros hombres en un contexto y en una situación espacio-temporal determinada y, desde allí, considerar al investigador como parte del mundo que estudia. Como señalan Hammersley y Atkinson, "Al incluir nuestro propio papel dentro del foco de investigación en el mundo que estamos estudiando, podemos desarrollar y comprobar la teoría sin tener que hacer llamamientos inútiles al empirismo, en su variedad naturalista o positivista" (1994, p. 40). Así, concebimos la reflexividad del investigador en el trabajo de campo a partir de la cual "describir una situación es construirla” (Vasilachis, 2007, p. 115). La reflexividad emerge como el soporte y a la vez la dinámica básica del planteo etnográfico, sustentada en la relación que se establece entre dos sujetos interactuando y participando. Así comprendida, la reflexión es una práctica de implicación y por tanto, ésta debe ser puesta en análisis en el proceso reflexivo mismo, aun cuando se trata de reflexionar sobre un objeto otro, otro sujeto, distinto de uno mismo (Souto, 2016).

La investigación narrativa es el contexto propicio para potenciar la reflexividad del investigador y utilizarla como metodología cualitativa potente para interpretar los datos de la investigación (Marques y Satriano, 2017). La narrativa se transforma en catalizador de los sentimientos, emociones, afecciones del propio investigador en el proceso del trabajo de campo. Narrar las propias sensaciones a medida que estas van sucediendo se transforma en un elemento potente de metacognición del proceso de investigación. Así, la narrativa regala la posibilidad de materializar y visibilizar, poner de manifiesto, la propia subjetividad del investigador social.

"Me sorprendió estar trabajando en la Biblioteca Nacional del Maestro y decir... soy Becario de Conicet y sentirme investigador. Lo más importante fue cuando encontré, así fortuitamente, en la sala de documentación el informe final de la Gestión de Edith Litwin en donde se detallaba el proyecto Polos de Desarrollo, objeto de mi tesis. La alegría me invadió como cuando a uno se le devela un mundo desconocido" (RA No42. Ciudad de Buenos Aires, 2903-2017)

"Hoy llegué a Buenos Aires para comenzar mi trabajo de campo. A las 8.30 estaba en la estación de Retiro. Nuevamente sentí lo mismo que en la primera vez que viajé a Rosario a iniciar mi doctorado. Ansiedad, temor, de no cumplir con la entrevista, preocupación para que el inicio sea prometedor y pueda tener buen trabajo de campo. Ahora recuerdo las palabras de Gessaghi (2016) cuando sostenía que el inicio del trabajo de campo suele ser angustiante porque solo al final podrás ver el trabajo realizado" (RA N². Ciudad de Buenos Aires, 30-112016)

Desde esta lógica, las investigaciones de corte narrativo no solo permiten que los actores investigados narren sus vidas resignificándolas desde el presente pero 
haciendo alusión a esa huella del pasado que marca (Gonzalez, 2017), sino que habilita al investigador mismo a poner en el lugar de sujeto narrador de su propia vida en relación a la investigación y al trabajo en campo. Es por ello que, como sostiene Guber "el conocimiento es revelado no al investigador, sino en el investigador, debiendo comparecer en el campo, debiendo reaprenderse y reaprender el mundo desde otra perspectiva" (2001, p. 54).

Utilizar instrumentos metodológicos como el diario autoetnográfico implica inscribirnos fuertemente en una perspectiva interpretativa sellada en las Ciencias Sociales a partir del llamado giro hermenéutico. Este paradigma privilegia el acceso al mundo de las interpretaciones (Geertz, 1994), restaurando el valor de las significaciones que otorgan los sujetos al mundo, en un esfuerzo por comprender las intenciones humanas (Bruner, 1988). Los textos construidos por los actores sociales, inevitablemente contextuales y complejos, se vuelven de este modo fuente de conocimientos relevantes como experiencia en el mundo, irreductibles a las explicaciones causales y a la vez exentos de las "garantías" de los métodos clásicos de las ciencias físicas y naturales (Bolívar, 2016).

Entendemos, en este contexto que las narrativas están presentes en todos los aspectos de la vida, abarcando desde las memorias personales hasta la literatura y la ciencia, la fotografía y el arte. No hay registros narrativos únicos, más bien son múltiples y polifónicos (Porta, 2015). Desde esta perspectiva, la narrativa combina el relato con el conocimiento y la comprensión de lo referido (Martin, 2008).

Siguiendo la senda hermenéutica trazada por Gadamer (1996), Ricoeur sostiene que la narrativa está íntimamente relacionada con el problema de la identidad personal y las aporías temporales (Ricoeur, 2009). Así, el estudio sobre la identidad personal y la identidad narrativa se presenta desde este autor como un trabajo filosófico reflexivo, de meditación, una actividad interpretativa. Es desde la narrativa donde se resuelven las paradojas de la identidad personal, "la persona entendida como un personal del relato no es una entidad distinta de sus experiencias, muy al contrario: comparte el régimen de la identidad dinámica propia de la historia narrada" (Ricoeur, 2006, p. 147).

Desde este mundo de significaciones, reflexionar sobre las implicancias del diario autoetnográfico como una de las técnicas metodológicas que utilizamos en nuestras investigaciones implica también reflexionar sobre el estudio mismo de la narrativa y sobre la propia subjetividad del investigador social.

De esta manera, como primera aproximación, podríamos decir que el diario autoetnográfico representa, justamente, la forma en que el investigador experimenta, siente, piensa y vive el proceso de investigación. El diario autoetnográfico es en sí mismo una narrativa. Narrativa producida por el sujeto que investiga a los efectos de poder explicitar las diversas vivencias, sentimientos, interpretaciones que va registrando y sintiendo a lo largo, no solo del proceso de investigación, sino, particularmente, en el mismo trabajo de campo. Es, como argumentan los autores, un relato personal del 
investigador en donde explicita su propia subjetividad. "La narrativización de la vida en un autorrelato, textualiza la vida, textualiza las experiencias vitales y las convierte en un texto" (Bolívar, Domingo, Fernández, 2001, p. 31).

Relatar o narrar las experiencias en el proceso de investigación es una forma de habitar nuestros propios sentidos y nuestras propias palabras (Gonzalez, 2017). Así, la escritura del diario autoetnográfico es "una forma en el espacio que prefigura el habitar de nuestras ideas" (Martínez y Bengoa, 2016, p. 47) a la vez que se transforma "en una aventura, llevada a cabo, en la ocupación del espacio" (Martínez y Bengoa, 2016, p. 48). Esta idea de habitar las significaciones personales es completada por Richarson cuando afirma que "las autoetnografías son altamente personalizadas, textos reveladores en los cuales los autores cuentan relatos sobre su propia experiencia vivida, relacionando lo personal con lo cultural" (2003, p. 512).

A continuación, los retazos muestran pinceladas de sentimientos y emociones que se ponen en juego en el habitar del trabajo de campo. Se destaca la figura del director de la investigación como guía, acompañante y maestro en el proceso,

"Simplemente tenía necesidad de escribir lo que estoy pensando y sintiendo luego de un enero de trabajo caluroso en Mar del Plata" [...] "Con el trabajo en capítulos de la tesis, me di cuenta como uno va madurando y metiéndose poco a poco en la tesis. Voy entendiendo procesos y armando en mi cabeza el esqueleto del documento final (la siento tan lejana y a la vez se vuelve poco a poco tan íntima)" (RA N¹9. Mar del Plata, 27-01-2017)

"Temo que me está costando soltar el campo, siento que siempre se puede buscar más, que me faltan datos. La figura del director se vuelve cada vez más importante, porque me marca los tiempos y los cierres. Ya es tiempo de soltar al campo y emprender la nueva etapa en la tesis" (RA N076. Ciudad de Buenos Aires, 1-06-2017)

"A las 15hs emprendí camino hacia la casa de quien fuera Asesora del Programa de Formación Docente que enmarcó el Proyecto Polos de Desarrollo. Pensar que hace unos años leía sus trabajos y ahora me encuentro yendo a la casa. Aquí aparece otra huella y aprendizaje. Recuerdo que mi director de tesis, contaba su costumbre de comprar masitas finas antes de visitar a un entrevistado. Es un gesto que crea el clima de cordialidad y confianza entre ambos sujetos de la investigación. En el camino hacia la casa de la Asesora, recordé el relato de mi director y dije ¿Por qué no hacerlo yo también? Huellas que surgen en el momento menos esperado. Así que pase por una panadería, compré $\square$ de masitas y me dirigí hacia el encuentro" (RA N073. Ciudad de Buenos Aires, 1-06-2017)

En el diario autoetnográfico, entendido como relato, el sujeto repiensa y reinventa sus experiencias en el campo, las interpreta tomando autoría de los hechos, y por tanto, pudiendo imaginar posibilidades de actuación futuras diferentes. Es en definitiva un registro de experiencias, un conocimiento consciente de la singularidad de cada individuo (Bolívar, Domingo, Fernández, 2001). A partir de narrar nuestras afecciones, aprendizajes y emociones, que en definitiva conforman nuestra propia subjetividad, nos transformamos en narradores de experiencias y prácticas de investigación (Marques y Satriano, 2017). Y así, toda narración autobiográfica ya supone en sí misma una interpretación, construcción y recreación de sentidos, lecturas del propio mundo de la propia vida. 
A diferencia de un diario de campo, el diario autoetnográfico que utilizamos en nuestras investigaciones recupera lo que el investigador va sintiendo, no sólo en el momento del trabajo de campo, sino a lo largo del proceso de investigación. Así, el diario se vuelve una suerte de bitácora en donde quedan registradas las emociones, ansiedades, preocupaciones, alegrías, desafíos, decisiones que va experimentando el investigador y que juntas implican una aproximación metacognitiva del proceso de investigación.

"Cuando terminé la entrevista con Elisa, me tomé el subte hacia Independencia y 9 de Julio. Allí me esperaba mi padre para emprender el viaje de vuelta a Mar del Plata. Como de costumbre, me tomé un café para esperarlo y mientras me dispuse a pasar la conversación del grabador a mi notebook y al Pendrive. Cuando prendo el grabador y lo enchufo a la notebook el mundo se detuvo por unos segundos. El grabador se puso en blanco y no respondió más. El archivo estaba adentro y no lo pude bajar [...] El viaje de vuelta fue difícil, mi ansiedad estaba a flor de piel. Pensaba permanentemente en las variantes que tenía, debía volver a hacer la entrevista, viajar de nuevo [...]. Llegando a Mar del Plata el grabador se apagó, se quedó sin batería. Pensé que cuando lo enchufe nuevamente iba a recuperar el archivo. Llegamos a las $23 \mathrm{hs,} \mathrm{lo}$ primero que hice fue verificar si andaba y ahí estaba el archivo. Felicidad y aprendizaje. Fue un aprendizaje enorme. Me sirvió para saber que tengo que confiar más. También entender que no todo depende de mí, ni de mi responsabilidad. He sido un afortunado en mi trabajo de campo, no he tenido mayores contratiempos. Tengo que relajarme más saber que los tiempos son distintos en la tesis y en la investigación. Aprendí a controlar, en parte mi ansiedad, y eso es bueno" (RA N0108. Ciudad de Buenos Aires, 3-08-2017)

"Si bien esta narrativa surge de mis emociones y experiencias y se vuelve sanadora y catalizadora, se vuelve potente cuando la hago dialogar con la escritura de la tesis. Entramar mi relato con los otros relatos provocando un solo registro polifónico se vuelve un desafío" (RA N¹28, Mar del Plata, 14/01/2018)

"Salí del Instituto con cierta nostalgia. Dejar el campo me genera cierto duelo o vacío. Nostalgia de haber cerrado una etapa. Siento que me involucre mucho. Fui uno más. Sentí que la teoría iba conmigo pero yo la utilizaba de acuerdo a los momentos. Hoy me doy cuenta que estaba preparado para el trabajo de campo. Aprendí mucho, no soy el mismo" (RA No99100, Esteban Echeverría, Bs. As, 15/06/2017)

A partir del registro autoetnográfico, el investigador puede reconstruir la investigación desde un lugar reflexivo, analizando el proceso desde otra magnitud. Lo que intentamos hacer a partir del diario autoetnográfico es tomar conciencia del proceso investigativo experimentado. El diario otorga la posibilidad de, una vez concluida la investigación, realizar la metacognición de la misma. Es de alguna manera la conciencia y la consideración por parte de la propia persona de sus estrategias y procesos cognitivos (Souto, 2016). Así, reflexionar sobre la acción realizada implica tanto la posibilidad de mejorar el propio aprendizaje como también la capacidad de conocer y mejorar las propias capacidades y limitaciones.

La tarea del investigador está atravesada por infinidad de tensiones a lo largo del proceso de investigación. Esas tensiones, en ocasiones, están relacionadas con las decisiones que se van tomando en conjunto con el director, en la elección y abordaje del tema y de la bibliografía, en la estructura del trabajo, en el trabajo de campo, en la interpretación de los datos obtenidos y particularmente en el trabajo de escritura final. 
El relato autoetnográfico constituye el ámbito fundamental para organizar la experiencia de la investigación, para exponer nuestras intuiciones a partir de los referentes empíricos que uno va relevando en el campo (Ellis, Adams y Bochner, 2015). Es, a la vez, una oportunidad para detectar sesgos personales, situaciones o vivencias que pueden de alguna u otra manera incidir en el mismo. La relación entre lo personal, lo emocional y lo intelectual se transforma mediante el análisis reflexivo que encuentra en el diario autoetnográfico el espacio propicio de su manifestación (Denzin, 2017). En dicha narración autoetnográfica el investigador objetiva momentos epifánicos (Ellis, Adams, y Bochner, 2015) del proceso de investigación vivido y cómo éstos "son tejidos a través de la multiplicidad de hebras de la subjetividad de la persona" (Denzin, 2017, p.85).

En este contexto, la autoreflexividad emerge como soporte y a la vez dinámica básica del planteo etnográfico, puesto que sin una verdadera introspección hacia las reflexividades del investigador no se puede alcanzar una interpretación compleja del objeto que se indaga. Es decir, toda situación que se observa se construye desde nuestra subjetividad. Explicitarla aporta no sólo una validación metodológica, sino que permite enriquecer la mirada y aceptar que nuestra subjetividad e implicación como sujetos está presente en la interpretación de los datos recogidos en campo. La reflexividad del investigador se transforma en el eje del trabajo etnográfico.

La narrativa y el enfoque narrativo vienen al auxilio del investigador etnográfico. Este enfoque permite al sujeto que investiga narrar sus sensaciones, miedos, angustias, alegrías, afecciones y emociones que va viviendo y experimentando a lo largo del trabajo de investigación, particularmente en la instancia del trabajo en terreno. Narrar nuestras experiencias y nuestras decisiones en campo permite obtener un nuevo instrumento de recolección de datos, pero a la vez implica utilizar esas mismas narraciones como elemento catalítico (Yedaide, Álvarez, Porta, 2015) del trabajo de investigación. La narrativa así se vuelve un bálsamo que nos regala la posibilidad de "encontrar la voz del corazón (...), la búsqueda de nuestra propia voz interior" (Nachmanovitch, 2014, p. 58). Nos permite, de alguna manera, objetivar nuestra propia subjetividad y al hacerlo tomamos conciencia también de nuestros procesos de formación como investigadores, de allí la validez catalítica de la narrativa (Anderson y Kerr, 2007).

En nuestro caso, a medida que avanzaba la investigación y nuestras visitas al campo, fuimos narrando, contando, autoreflexionando nuestras vivencias y experiencias en un diario autoetnográfico. Fuimos documentando narrativamente todo lo que sentimos, vivimos, fuimos y somos en torno al proceso de investigación. En dicho registro, entendido como relato, repensamos y reinventamos nuestras sensaciones, las interpretamos tomando autoría de los hechos, y por tanto, pudimos imaginar posibilidades de actuación futuras. Es, en definitiva, un registro de experiencias, un conocimiento consciente de la singularidad de cada individuo (Bolivar, Domingo, Fernández, 2001).

Desde el punto de vista metodológico, hemos utilizado el registro escrito para dar cuenta de lo que fuimos viviendo a lo largo del proceso de investigación. El diario se fue completando a medida que habitábamos cada vez más los territorios del trabajo 
de campo. Entrevistas, grupos focales, visitas a Institutos Formadores de Docentes, viajes, encuentros, conversaciones con colegas. Todo fue registrado a partir de nuestra vivencia y sentimientos. El texto construido fue resignificándose con el tiempo y fue constituyéndose como una valija de experiencias altamente formativas y profundamente subjetivante.

\section{Conclusiones que invitan al debate: La narrativa autoetnográfica como magma de significaciones y cristalización de sentidos.}

Hemos intentado, en este artículo, habilitar la reflexión en torno a la implicación y a las sensibilidades que se ponen en juego por parte del investigador a lo largo del proceso de investigación, particularmente en la instancia del trabajo de campo. Implicación, reflexión y narración han sido los conceptos que vertebraron el trabajo y que configuran los elementos centrales si se pretende sincerar los procesos epifánicos y vitales que van aconteciendo en la persona del investigador a lo largo del proceso de investigación. Al caracterizar la narrativa autoetnográfica como magma de significaciones (Porta, 2017) hacemos referencia a la polifonía de significados que el narrador va entretejiendo en la medida que narra sus propias experiencias. En el relato se produce, inevitablemente, la recursividad de los significados que el investigador le otorga a sus interpretaciones y vivencias. Como sostiene Blanco, en esta recursividad metacognitiva "la autoetnografía se transforma en una forma narrativa de producir conocimientos" (2012, p.49).

En el ir y venir de la interioridad a la palabra escrita, de lo vivido al recuerdo, de volver hacia nuestros sentimientos desde otro lugar una y otra vez hace que el trabajo de escribir, de narrar forme, eduque (Souto, 2016). Quien narra sus experiencias, sus emociones, sus miedos, afecciones y alegrías puede abrir su pensar y su propia subjetividad y así aprender de lo ya vivido, resignificándolo, hallándole nuevos sentidos.

Partir de la aceptación de que el sujeto que investiga se implica y al implicarse modifica el entorno y su propia reflexividad en relación al objeto de estudio requiere la necesaria explicitación de todo ese camino que recorre el investigador. De esta manera, la narrativa se ubica en el lugar donde "la existencia humana toma forma, donde se elabora y se experimenta bajo forma de una historia" (Ricoeur, 2009, p. 29).

El escribir sobre uno mismo adquiere significados fuertes en nuestra cultura (González, 2017). Lo autobiográfico narrativo ayuda a construir la existencia de un sujeto autor de sí mismo, que se inscribe en la sociedad desde este lugar (Souto, 2016). Los relatos y registros autobiográficos permiten conocerse más a sí mismo, conocer más a los otros, otros que nos miran, nos reflejan y nos subjetivan. Aquí radica la potencia del diario autoetnográfico en la investigación cualitativa-narrativa. El registro, además de ser una pieza metodológica validada e importante a los efectos de reconstruir el campo y lo que sucede allí, es potente en relación al relato propio del investigador que se configura en auto-narración subjetivante. Nosotros, como investigadores, no escapamos de esa implicación señalada con anterioridad. Manifestarla y analizarla se vuelve indispensable para la investigación misma, puesto que se explicitan los propios procesos vitales del 
sujeto que interpreta al objeto de estudio.

Probablemente, en el seno de la académica tradicional y la epistemología clásica, la manifestación de las emociones, afecciones, deseos y temores mediante relatos de experiencias personales no tenga un lugar destacado. Pero para aquellas epistemologías narrativas otras (Walsh, 2014; De Souza Santos, 2016) que se posicionan en formas alternativas de producir conocimiento, recuperar las voces y las subjetividades de los actores inmersos en los procesos de investigación se torna altamente potente y extremadamente enriquecedor. La inclusión de la subjetividad y del sujeto es rasgo predominante en lo narrativo, de allí la incorporación de este enfoque en nuestras investigaciones (Bolívar, 2016). De esta forma, documentar narrativamente lo que somos y lo que hacemos se vuelve nodal para habitar nuevos territorios metodológicos y epistemológicos.

Para concluir el artículo, elegimos una narrativa que justamente define nuestro trabajo de campo como ese salto al vacío que solo al final del proceso podremos articular en su totalidad. Pero al mismo tiempo, jugarse por metodologías otras también implica un salto hacia lo desconocido, pero con la serena certeza de que ese camino deja huellas altamente formativas en nuestro ser como investigadores y como personas.

"Creo que no me doy cuenta de los pequeños pasos que voy dando porque siempre la mirada está puesta en el futuro, pero hoy que estoy próximo a concluir mi trabajo de campo siento que he crecido y que ha sido un tiempo de profundo aprendizaje y conocimiento de mí mismo. En una clase explicité que el trabajo de campo es un salto al vacío. Una colega que participaba de ese taller de tesis me envió un mensaje diciendo...con tus palabras recordé la pintura de Yves Klein, un salto al vacío, gracias por el recuerdo y el testimonio. Esas son cosas que a uno lo marcan". (RA N¹02. Mar del Plata, 3-08-2017)

\section{Referencias Bibliográficas}

Agar, M. (2006), "An Ethnography By Any Other Name", Forum: Qualitative Social Research, 7(4), Recuperado de http://nbnresolving.de/urn:nbn:de:0114-fqs0604367

Aguirre, J. (2017) Voces territorializadas y sentidos vitales de una política pública innovadora en la formación docente argentina. Journal for Educators, Teachers \& Trainers. (9)1, 253-266. http://jett.labosfor.com/index.php/jett/article/view/310/322

Alvarez, Z; Porta L. Sarasa M. (2010) La investigación narrativa en la enseñanza: las buenas prácticas y las biografías de los profesores memorables. Revista de Educación. 1(1), 159-179. Recuperado de http://fh.mdp.edu.ar/revistas/ index.php/r_educ/article /view/12

Anderson, G. y Herr, K. (2007) El docente-investigador: la investigación acción como una forma válida de generación de conocimientos, en: Sverdlick, I. (Comp.), La investigación educativa. Una herramienta de conocimiento y acción. (pp. 115-135) Buenos 
Aires: Noveduc.

Blanco, M. (2012) Autoetnografía: una forma narrativa de generación de conocimientos. Andamios, (9)19, 49-74. Recuperado de https://andamios.uacm.edu.mx/index. php /andamios/article/view/390

Bolívar, A., J. Domingo y M. Fernández. (2001). La investigación biográfico-narrativa en educación. Enfoque y metodología. Madrid: La Muralla

Bolivar, A. (2016) Conjugar lo personal y lo político en la investigación (auto)biográfica: Nuevas dimensiones en la política educacional. Revista Internacional de Educación Superior. (2)2, 341-365. Recuperado de https://periodicos.sbu.unicamp. br/ojs/index. php /riesup/article/view/8650555

Bruner, J. (1998). Realidad mental, mundos posibles. Barcelona: Gedisa.

Denzin, N. y Lincoln Y. (2015). Manual de investigación cualitativa, IV: métodos de recolección y análisis de datos. México: Gedisa.

Denzin, N. (2017) Autoetnografía Interpretativa. Investigación cualitativa, 2(1), 81-90. http://dx.doi.org/10.23935/2016/01036.

De Sousa Santas, B. (2016) Epistemologies of the South and the future. From the european south. 1(1), 17-29. Recuperado de http://europeansouth.postcolonialitalia.it/ archive

Ellis, C. Adams, T. y Bochner, A. (2015). Autoetnografía, un panorama. Astrolabio, nueva época. 14(1), 249-273. Recuperado de https://revistas.unc.edu.ar/index.php/ astrolabio/article/viewFile/11626/12041

Fernández, L. (2010). La investigación institucional de organizaciones educativas. Algunos aportes de estudios realizados en el Programa "Instituciones educativas de UBA". Praxis Educativa. 14(14). 66-76. http://cerac.unlpam.edu.ar/index.php/praxis/article view/427/372

Gadamer, H. (1996). Verdad y método. Salamanca: Sígueme.

Geertz, C (1994) Conocimiento local, ensayo sobre la interpretación de la cultura. Madrid. Paidós.

Gessaghi, V (2016) La educación de la clase alta argentina. Buenos Aires. Siglo XXI

Gonzalez, M.F. (2017) "Las narrativas autobiográficas en la construcción de la experiencia y el sí mismo". Linhas Críticas, 23 (51), 428-448. Recuperado de http://periodicos. unb.br/index.php/linhascriticas/article/view/28460/20073 
Goodson, I. (2017) International Handbook on Narrative and Life History. New York: Routledge.

Guber, R. (2001). La Etnografia: Método, Campo y Reflexividad. Buenos Aires, Norma

Guerrero Muñoz, J. (2014) El valor de la autoetnografía como fuente para la investigación social: del método a la narrativa. Azarbe. 1(3), 237-242. Recuperado de http:// revistas.um.es/azarbe/article/view/198691

Gvirtz, S y Torre, E. (2015). La dimensión política técnica de la gestión educativa. En: Tedesco, J.C. (comp) La Educación Argentina hoy. La urgencia del largo plazo. (pp. 3960) Buenos Aires: Siglo XXI.

Hammersley, M y Atkinson, P. (1994) Etnografia y método de investigación. Barcelona: Paidós.

Marques, V. y Satriano, C. (2017). "Narrativa autobiográfica do próprio pesquisador como fonte e ferramenta de pesquisa" Linhas Críticas, 23 (51), 369-386. Recuperado de http://periodicos.unb.br/index.php/linhascriticas/article/view/28457/20070

Martin, V. (2008). A narrative inquiry into the effects of serious illness and major surgery on conceptions of self and life story. PhD Thesis. Bristol: University of Bristo

Martínez, C y Bengoa G. (2016) Habitares; los croquis de la palabra. Mar del Plata: Eudem.

Nachmanovitch, S. (2014). Free Play. La improvisación en la vida y en el arte. Buenos Aires: Paidós.

Noy, C. (2003) "La escritura de transición: Reflexiones en torno a la composición de una disertación doctoral en metodología narrativa" Forum: Qualitative Social Research. (4)2, 1-30 Recuperado de http://www.qualitative-research.net/index.php/fqs/ rt/printer

Porta, L. (2015) Narrativas sobre la enseñanza en torno a la "didáctica de autor". Las maravillas cotidianas que abren a la percepción en el aula universitaria. Revista del IICE. 1(37) 41-54. Recuperado de http://revistascientificas.filo.uba.ar/index.php/iice/ article/view/3449/3183

Porta, L. (2017) La narrativa biográfica como magma de significaciones y cristalización de sentidos. Conferencia de Apertura de las II Fábrica de Ideas (Historias y Prácticas). Facultad de Humanidades- UNMdP, Mar del Plata.

Porta, L. y Aguirre, J. (2017) Las principales políticas públicas de Formación Docente desde las voces de los actores. El caso de los Polos de Desarrollo (2000-2001). Archivos de Ciencias de la Educación. 11(12). 1-17. Recuperado de http://www.archivos deciencias.fahce.unlp.edu.ar/article/view/Archivose033 
Porta L. y Yedaide, M. (2017) Pedagogía(s) Vital(es). Cartografías del pensamiento y gestos ético-políticos en perspectiva descolonial. Mar del Plata: EUDEM.

Porta, L. Aguirre, J y Bazan, S. (2017) La práctica docente en los profesores memorables. Reflexividad, Narrativa y sentidos vitales. Diálogos Pedagógicos. 16(30), 15-36. Recuperado de http://dx.doi.org/10.22529/dp.2017.15(30)01

Richardson, L. (2003), Writing. A Method of Inquiry, en Denzin, N. y Lincoln, Y. (eds.), Collecting and Interpreting Qualitative Materials, Thousand Oaks, California: Sage.

Ricoeur, P. (2006) Si mismo como otro. México: Siglo XXI

Rockwell, E. (2009) La experiencia etnográfica. Buenos Aires: Paidós

Sanjurjo, L. (2002) La formación práctica de los docentes. Reflexión y acción en el aula. Rosario: Homo Sapiens.

Souto, M. (2016) Pliegues de la formación. Sentidos y herramientas para la formación docente. Rosario: HomoSapiens.

Stoller, P. (1999), "Back to the ethnographic future", Journal of Contemporary Ethngraphy, Thousand Oaks, California: Sage

Suárez, D. (2017). Docentes, relatos de experiencia y saberes pedagógicos: La documentación narrativa de experiencias en la escuela. Investigación Cualitativa, 2(1), 48-60. doi:http://dx.doi.org/10.23935/2016/01034

Taylor, S. y Bogdan, R. (2007). Introducción a los métodos cualitativos de investigación. Buenos Aires: Paidós.

Vasilachis, I. (2007) Estrategias de investigación cualitativa. Buenos Aires: Gedisa

Walsh, C. (2014) Pedagogías decoloniales caminando y preguntando: notas a Paulo Freire desde Abya Yala, Entramados, 1(1), 17-31. Recuperado de http://fh.mdp.edu. ar/revistas/index.php/entramados/article/view/1075

Yedaide, M. M., Alvarez, Z., y Porta, L. (2015). La investigación narrativa como moción epistémico-política. Revista Científica Guillermo de Ockham, 13(1), 27-35. Recupera do de http://www.redalyc.org/articulo.oa?id=105342821002

\section{Documentos Gubernamentales.}

Argentina. Ministerio de Cultura y Educación de la Nación. 2001. Programa Nacional de Formación Docente (2001) Informe de Gestión. Recuperado de http://www.bnm. me.gov .ar/giga1/documentos/EL005496.pdf 


\section{Biografia}

\section{Jonathan Aguirre}

Especialista en Docencia Universitaria por la Universidad Nacional de Mar del Plata (UNMdP). Becario Interno Doctoral del Consejo Nacional de Investigaciones Científicas y Técnicas (CONICET). Doctorando en Humanidades y Artes con mención en Ciencias de la Educación por la Universidad Nacional de Rosario (UNR). Profesor en Historia (UNMdP). Docente e Investigador del Departamento de Ciencias de la Educación. Centro de Investigaciones Multidisciplinarias en Educación (CIMED) de la Facultad de Humanidades de la Universidad Nacional de Mar del Plata (UNMdP).

ORCID: https://orcid.org/0000-0002-6291-2545

\section{Luis Porta}

Doctor en Filosofía y Ciencias de la Educación por la Universidad de Granada (España). Docente e Investigador (categoría I) del Departamento de Ciencias de la Educación de la Facultad de Humanidades (UNMdP) e Investigador Independiente del Consejo Nacional de Investigaciones Científicas y Técanicas (CONICET). Profesor Titular Regular con dedicación exclusiva de la Cátedra Problemática Educativa. Director del Centro de Investigaciones Multidisciplinarias en Educación (CIMed- UNMdP). Director de la carrera de posgrado Especialización en Docencia Universitaria (UNMdP) y del "Grupo de Investigaciones en Educación y Estudios Culturales" (GIEEC- UNMdP). 